\title{
L'uso della toponomastica come indicatore di insediamenti e strutture fortificate: il caso toscano
}

The use of toponymy as an indicator of settlements and fortified structures: the Tuscan case

\author{
Camillo Berti ${ }^{\text {a }}$, Massimiliano Grava ${ }^{\text {b }}$ \\ ${ }^{a}$ Università degli Studi di Firenze, Florence, Italy, camillo.berti@unifi.it \\ ${ }^{\mathrm{b}}$ Università di Pisa, Pisa, Italy, massimiliano.grava@unipi.it
}

\begin{abstract}
The purpose of this contribution is to analyze the spatial distribution of the place names referred to the Tuscan territory, to fortified structures and settlements, through the study of the place names recorded geodatabase RE.TO.RE. (Regional Toponymic Repertory) created by the Tuscany Region with the scientific contribution of the Universities of Pisa, Florence and Siena. The Tuscan toponyms has been the object of both a synchronic study within each of the cartographic sources that make up the geographical database, and a diachronic analysis between the temporal thresholds in which the archive is articulated. The database, extrapolated from cartographic supports, in fact covers a time span between the first decades of the nineteenth century (nineteenth century land registries) and the most recent information series produced in the regional context (Carta Tecnica Regionale). In the contribution, the place names related in various ways to different types of structures and fortified settlements, such as castle, fort, tower, fortress, has been analyzed both in relation to the distribution and spatial aspects, and in reference to their evolutionary dynamics (persistence, disappearance, transformation), with the aim of identifying possible relationships between the territory and the distribution in time and space of the different types of fortifications. From a methodological point of view, the study has been carried out, in addition to the traditional tools of the topomastic survey, especially taking advantage of the potential of spatial analysis functions typical of geographical information systems.
\end{abstract}

Keywords: Place names/Toponymy, fortifications, Tuscany, GIS analysis.

\section{Introduzione}

Lo scopo di questo contributo è analizzare la distribuzione spaziale e l'evoluzione temporale nel corso degli ultimi due secoli dei nomi di luogo riferiti, per il territorio toscano, a strutture e insediamenti fortificati, attraverso lo studio dei toponimi censiti nella banca dati geografica RE.TO.RE. (Repertorio Toponomastico Regionale) realizzata dalla Regione Toscana con il contributo scientifico delle Università di Pisa, Firenze e Siena.
I toponimi toscani sono stati oggetto sia di uno studio sincronico all'interno di ciascuna delle fonti cartografiche che compongono il database geografico, sia di un'analisi diacronica tra le soglie temporali in cui l'archivio è articolato. La banca dati, estrapolata da supporti cartografici, copre infatti un arco temporale compreso tra $\mathrm{i}$ primi decenni del XIX secolo (catasti particellari ottocenteschi) e le più recenti serie informative prodotte in ambito regionale (Carta Tecnica Regionale). 
Nel contributo, i nomi di luogo correlati in vario modo a diverse tipologie di strutture e insediamenti fortificati, come castello, forte, torre, rocca, fortezza, sono stati analizzati sia in relazione agli aspetti distributivi e spaziali, sia in riferimento alle loro dinamiche evolutive (persistenza, scomparsa, trasformazione), con l'obiettivo di individuare eventuali relazioni tra il territorio e la distribuzione nel tempo e nello spazio delle diverse tipologie di fortificazioni.

Dal punto di vista metodologico, lo studio è stato effettuato, oltre che attraverso gli strumenti tradizionali dell'indagine toponomastica, soprattutto sfruttando le potenzialità delle funzioni di analisi spaziale proprie dei sistemi informativi geografici.

\section{Il geodatabase}

Il Repertorio Toponomastico Regionale, realizzato con il supporto di applicativi GIS, è stato costituito impiegando quattro basi cartografiche che coprono un arco temporale che va dalla prima metà del XIX secolo fino ai giorni nostri. A tal fine sono state utilizzate: le mappe dei catasti storici degli Stati preunitari toscani (scale 1:2000-5000); le mappe nelle diverse edizioni 1:25000 dell'Istituto Geografico Militare; le odierne mappe catastali gestite dalla Agenzia delle Entrate e la Carta Tecnica Regionale, nelle sue due edizioni (1:2000 e 1:10000).

L'implementazione delle informazioni toponomastiche all'interno della banca dati dei nomi di luogo della Carta Tecnica Regionale (CTR) ha dunque preso avvio con l'acquisizione dei toponimi presenti nelle mappe ottocentesche, provenienti dall'archivio digitale denominato CA.STO.RE. (CAtasti STOrici REgionali). Queste immagini, conservate nei diversi Archivi di Stato delle province toscane, costituiscono un patrimonio cartografico di oltre dodicimila elementi, schedati e georeferenziati, che comprendono Quadri d'unione, Fogli mappali e Sviluppi. La banca dati contiene le mappe dei seguenti catasti preunitari:

- Catasto Napoleonico, iniziato dai Francesi durante l'annessione della Toscana all'Impero tra il 1808 e il 1814 ;
- Catasto Generale di Terraferma (anche detto Lorenese o Ferdinandeo-Leopoldino), realizzato durante il governo dei Granduchi di Toscana Ferdinando III e Leopoldo II di Lorena, intrapreso nel 1817 e attivato nel 1835;

- Catasto delle Isole dell'Arcipelago Toscano, per le quali l'accatastamento fu realizzato successivamente all'attivazione di quello di terraferma, tra il 1840 ed il 1845 ;

- Catasto Borbonico, attinente al territorio del Ducato di Lucca, ordinato nel 1829 dal Duca Carlo Lodovico di Borbone e terminato dai Savoia nel 1869;

- Catasto Estense del Ducato di Massa e Carrara, avviato con decreto della Duchessa Maria Beatrice d'Este nel 1820, reso esecutivo nel 1824 e rimasto attivo fino alla metà del XX secolo.

L'archivio digitale RE.TO.RE., realizzato integrando i toponimi esistenti nella CTR con l'acquisizione dei toponimi presenti nella basi cartografiche precedentemente citate, costituisce quindi una banca dati di notevole importanza per la toponomastica toscana, tanto più per il fatto che è stata resa disponibile in forma gratuita (con licenza CC-BY) tramite il portale cartografico della Regione Toscana, sia per la consultazione (tramite webGIS o WMS), sia per il download dell'intero archivio in formato shapefile (http://www.regione.toscana.it/-/geoscopio).

\section{Analisi dei dati}

Da questo geodatabase, tramite opportune operazioni di interrogazione, è stato possibile estrarre i toponimi riferibili ad alcune tipologie di strutture fortificate presenti sul territorio toscano tra il XIX e l'inizio del XXI secolo. Nell'ambito di questo lavoro, che sarà oggetto di successivi approfondimenti, si è fatto riferimento ai termini che ci sono apparsi più significativi: castello, forte/fortezza, torre, rocca. È opportuno precisare che si sono presi in considerazione nomi di luogo riferibili direttamente a strutture fortificate (ad esempio, Castello di Brolio, Forte Stella), mentre non si è tenuto conto dei toponimi semanticamente connessi a strutture fortificate (Castiglion Fiorentino; Rocca San Casciano). Pertanto, in questo contesto, non si è tenuto con- 
to della distinzione tra toponimi veri e propri e i cosiddetti "pseudo-toponimi" (Cantile, 2004), cioè le denominazioni di particolari categorie di oggetti geografici, riportati nelle carte a corredo dei relativi simboli (ad esempio, "pozzo", "sorgente", "rudere", etc.).

Grazie a specifiche query è stato possibile analizzare tanto le variazioni di densità dei toponimi relativi alle diverse tipologie di manufatti, quanto l'evoluzione diacronica nell'arco temporale coperto dalla banca dati. Sono state, inoltre, realizzate carte tematiche a simboli puntuali, finalizzate alla lettura geografica della distribuzione dei toponimi riferibili a sistemi di difesa (Fig. 1). Attraverso funzioni di analisi spaziale proprie dei sistemi informativi geografici, sono state anche elaborate carte di densità, per le quali si è scelto di utilizzare algoritmi kernel, calcolando una superficie circolare di $4000 \mathrm{~m}$ intorno ai toponimi, distanza a nostro avviso ritenuta la più idonea per definire, ad una scala regionale, un chiaro inquadramento del fenomeno di concentrazione (non presentate in questo contributo per motivi di spazio).

\begin{tabular}{lr} 
Toponimo & Numero di entità \\
\hline Bastion* & 49 \\
\hline Rocca* & 275 \\
\hline Torr* & 1367 \\
\hline Castell* & 1521 \\
\hline Fort* & 176 \\
Totale & 3388 \\
\hline
\end{tabular}

Tab. 1. Numero di toponimi riferibili a installazioni militari presenti nel DBT.

Complessivamente, sono selezionati nel territorio toscano quasi 3400 toponimi relativi a strutture con funzione strategica, ma la ripartizione tra i vari termini mostra una netta prevalenza, rispetto alle altre prese in considerazione, delle voci torr* e castell* e (Tab. 1), che rappresentano rispettivamente il 40 e il $45 \%$ del totale.

L'analisi geografica mostra una distribuzione spaziale complessiva piuttosto omogenea della toponomastica riferita a strutture "militari", pur notando una maggiore densità di toponimi nella Toscana centro-settentrionale, verosimilmente imputabile al minore popolamento della parte meridionale della regione (Fig. 1). Tuttavia, analizzando separatamente la localizzazione dei diversi nomi si può invece osservare come i differenti tipi di fortificazioni si caratterizzino per una distribuzione particolare, che probabilmente è da mettere in relazione con le funzioni originarie delle strutture che i toponimi descrivono: mantenendo nel corso del tempo la medesima ubicazione, come è noto, i nomi di luogo, in molti casi, costituiscono una preziosa testimonianza dell' assetto del territorio nel passato.

Particolarmente significativo, tra quelli che abbiamo considerato, appare il caso dei toponimi Torr* che presentano una notevole concentrazione nell'area dell'attuale provincia di Firenze, mentre nella parte più meridionale della Toscana si trovano ubicati lungo la fascia costiera della Maremma. Presentano un pattern distributivo simile i toponimi caratterizzati dal prefisso Fort*, comprendenti termini come "forte", "fortino", "fortezza": anche in questo caso si nota una maggiore presenza nelle zone interne della Maremma, in passato scarsamente abitate, ove era quindi indispensabile installare infrastrutture per il controllo del territorio. Nel caso dei nomi di luogo Castell* e Rocca* la distribuzione dei toponimi risulta relativamente omogenea su tutto il territorio regionale, specie per quanto riguarda i castelli, fatta eccezione per una piccola area di concentrazione nel Pistoiese. Riguardo al vocabolo Rocca* è da notare che i toponimi attestati si trovano localizzati in piccoli gruppi, attestati in tutte le aree del territorio regionale ad eccezione della provincia di Siena. Limitate numericamente e prevalentemente attestato in ambito urbano, il termine Bastion*, che semanticamente descrive una parte specifica della struttura fortificata.

Un tentativo di analisi della densità toponomastica può essere effettuato elaborando i dati del Repertorio Toponomastico Regionale in ambiente GIS tramite funzioni di spatial analysis di tipo kernel density. Come si può osservare nella Fig. 2, l'area caratterizzata dalla massima densità corrisponde alla media e bassa valle dell'Arno, che è anche -sia recentemente che è in passatola zona più popolata della regione. Altre aree 


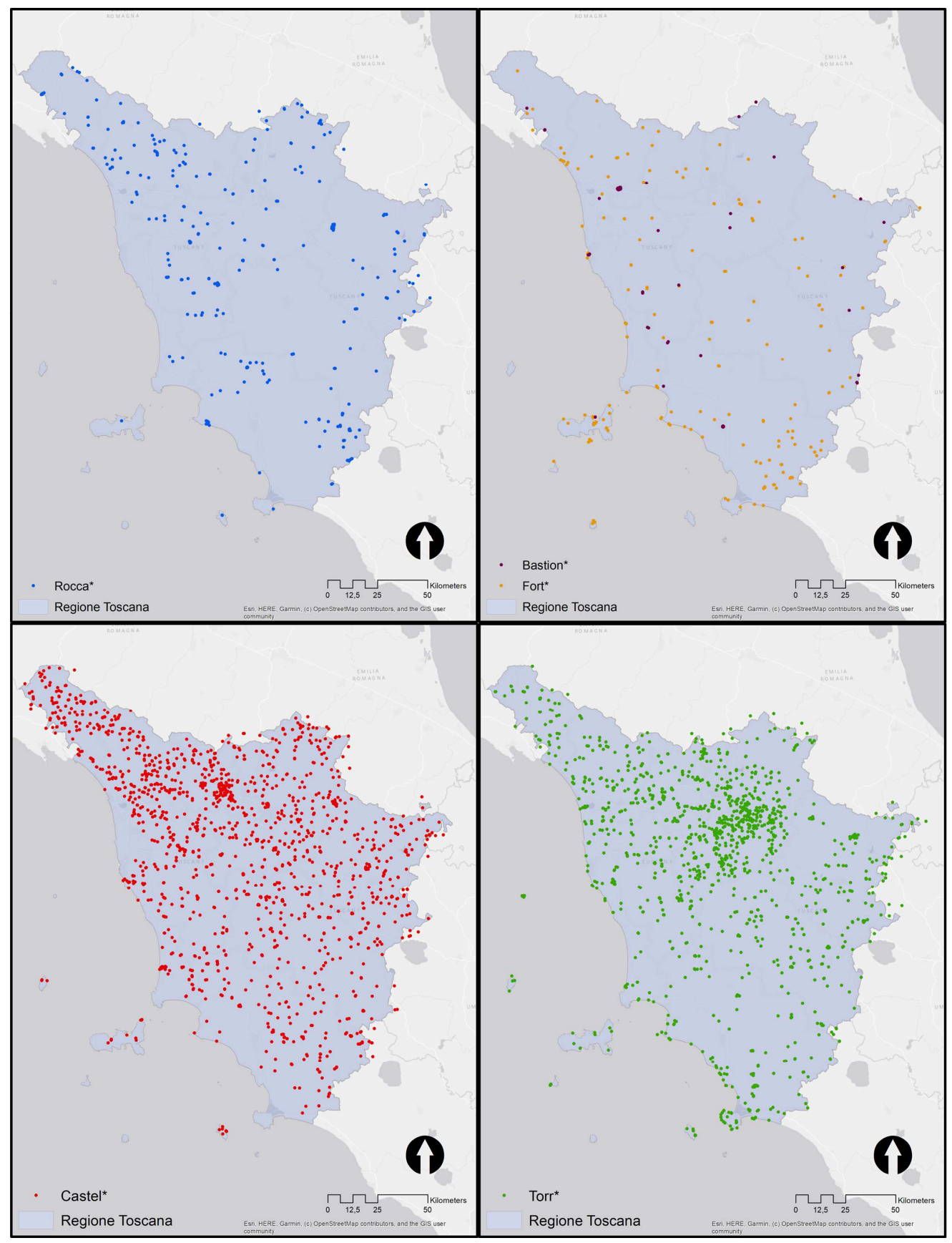

Fig. 1. Distribuzione spaziale dei nomi di luogo riferibili a strutture fortificate: castell*, torr*, fort*, bastion* e rocca* (elaborazione degli autori su dati Regione Toscana, REpertorio TOponomastico REgionale). 


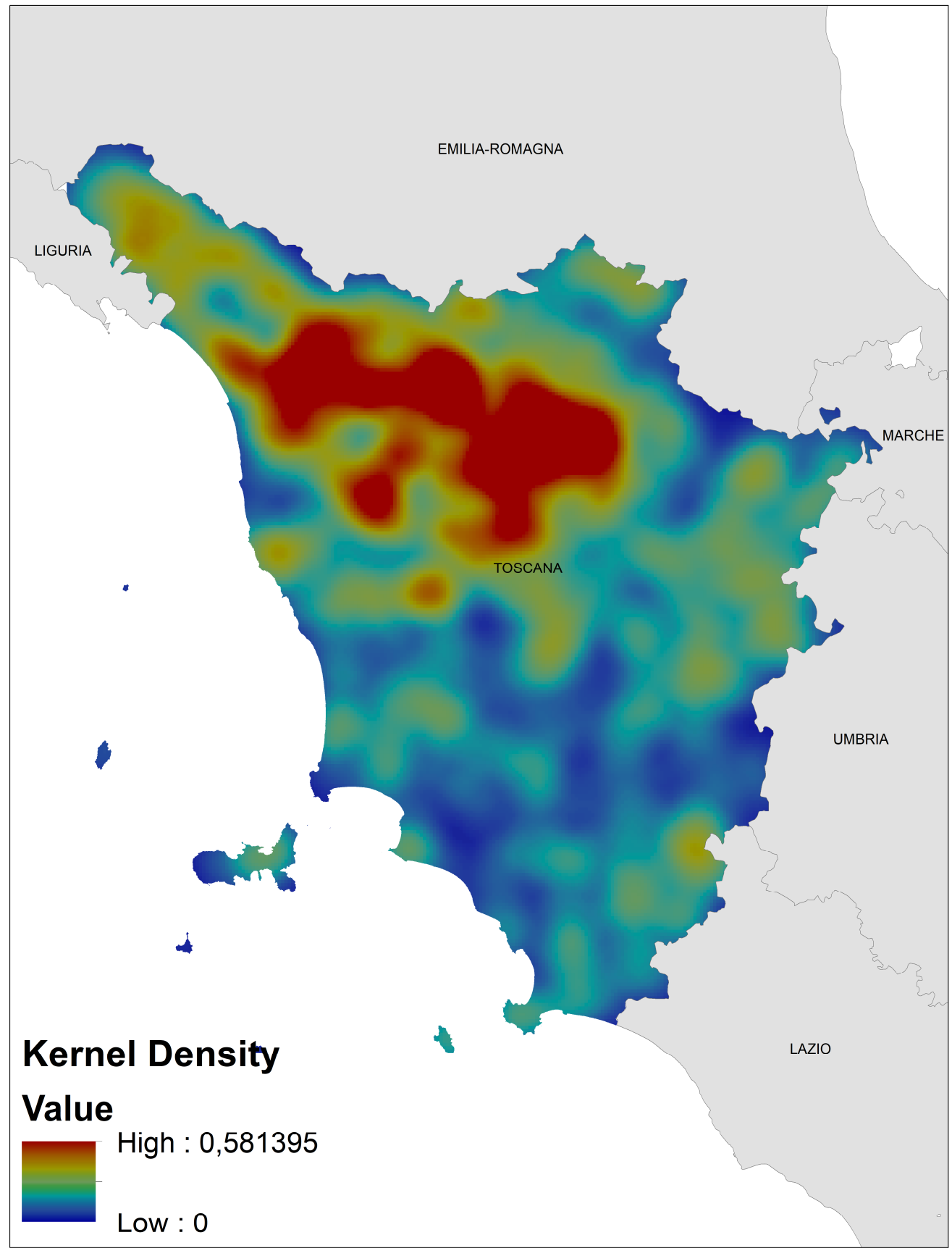

Fig. 2. Carta della densità dei toponimi considerati, elaborata con algoritmo Kernel Density con raggio circolare di 1 $\mathrm{km}$ impiegando i dati puntuali selezionati nella banca dati topografica RE.TO.RE. (elaborazione degli autori su dati Regione Toscana). 
dove si notano concentrazioni significative sono rappresentate dalle subregioni corrispondenti ad alcuni bacini intermontani appenninici (Garfagnana, Lunigiana, Casentino, Valdichiana) e ad alcuni gruppi montuosi, come l'Amiata e le Colline Metallifere.

Analizzando il numero di toponimi presenti nelle province attuali, si osserva che il territorio con il maggior numero di riferimenti a infrastrutture militari sia quello di Firenze con 782 ricorrenze, seguito da Prato (441), Grosseto (432), Lucca (383) e Siena (328). Minore il numero di toponimi delle provincie di Pisa, Livorno, Massa Carrara e Pistoia.

Dal confronto del numero di attestazioni presenti nelle diverse banche dati per ciascuna voce non risultano variazioni significative: il maggior numero di toponimi è riportato nella carta tecnica regionale e, comunque, per tutti i termini considerati si registrano variazioni comparabili proporzionalmente. È pertanto lecito ipotizzare che tali differenze numeriche siano da imputare più alle caratteristiche della rilevazione che non ad un'evoluzione temporale della presenza dei manufatti descritti dai toponimi stessi (Tab. 2).

\begin{tabular}{lrrr} 
Toponimo & CA.STO.RE. & Catasto & CTR \\
\hline Bastion* & 22 & 11 & 29 \\
\hline Rocca* & 132 & 130 & 153 \\
\hline Torr* & 721 & 756 & 763 \\
\hline Castell* & 721 & 783 & 875 \\
\hline Fort* & 80 & 76 & 90 \\
\hline
\end{tabular}

Tab. 2. Numero di toponimi riferibili a installazioni militari attestati nelle diverse banche dati che compongono il Repertorio Toponomastico Regionale.

\section{Conclusioni}

Complessivamente, lo studio effettuato ha messo in risalto l'importanza di poter disporre di base toponomastica come l'archivio digitale RE.TO.RE., che integrando quattro differenti fonti cartografiche, consente di eseguire sia analisi sincroniche, che diacroniche. Sebbene la ricerca meriti ulteriori approfondimenti, lo studio della distribuzione spaziale dei nomi di luogo riferibili alle strutture difensive/militari, pur tenendo conto delle complesse problematiche legate all'utilizzo della toponomastica, sembra possa fornire un contributo interessante anche all'analisi geografica di tali strutture. In particolare, è stato possibile evidenziare la distribuzione relativamente omogenea e la correlazione con la densità di popolazione per termini come castell* e rocca*, rispetto alla particolare localizzazione di altri nomi di luogo, come, ad esempio, fort*, che si presenta attestato lungo tutto il litorale, evidenziando la correlazione di questo termine con una specifica funzione difensiva.

\section{Bibliography}

Arcamone, M.G. (1981). "Antroponimia germanica nella toponomastica italiana", in La toponomastica come fonte di conoscenza storica e linguistica, Giardini, Pisa.

Bailey, T.J.; Schick, J.B.M. (2009). "Historical GIS: Enabling the Collision of History and Geography", Social Science Computer Review, 27, 3.

Berti, C.; Cassi, L.; Zamperlin, P. (2018). "Lama, pantano, padule. Toponomastica storica delle aree umide toscane", in Atti della 22 a Conferenza ASITA (Bolzano, 27-29 novembre 2018), Milano, ASITA, pp. 141-150. 
Campana, S. (2003). "Catasto leopoldino e GIS Technology: metodologie, limiti e potenzialità", in Trame nello spazio. Quaderni di geografia storica e quantitativa, Laboratorio Informatico di Geografia, 1, Siena.

Cantile, A. (2004), "Norme toponomastiche nazionali”, in Atlante dei tipi geografici, Firenze, pp. 83-89.

Cassi, L. (2015). Nomi e Carte. Sulla toponomastica della Toscana, Pacini, Pisa.

Cassi, L.; Marcaccini, P. (1991). "Appunti per la revisione della toponomastica nella cartografia a grande scala. Saggio di correzione ed integrazione di un elemento della carta tecnica regionale 1:5000 della Toscana", GEOGRAFIA, 2-3, pp. 100-110.

Cassi, L.; Marcaccini, P. (1992). "Gli «indicatori geografici» per la schedatura toponomastica. Criteri e norme per la loro definizione", GEOGRAFIA, 2-3, pp. 92-102.

Cassi, L. (2006). "Il contributo della geografia alle raccolte toponomastiche", in Toponomastica e beni culturali. Problemi e prospettive, Società storica della Valdelsa, San Gimignano. pp. 105-113.

Cassi, L. (2004). "Toponomastica", in Associazione dei Geografi Italiani. Progetti di ricerca dei Gruppi di lavoro, Patron Ed., Bologna, pp. 43-48.

Gregory, I.; Healey, R.G. (2007), "Historical GIS: structuring, mapping and analyzing geographies of the past", Progress in Human Geography, 31, 5.

Grava, M. ; Maestro, F. Del; Flora, V.; Gabellieri, N.; Gesualdi, M.; Lucchesi, F.; Martinelli, A.; Tarchi, G.; Tofanelli, M.; Biagioli, G. (2013). "Un patrimonio da salvare: toponomastica e microtoponomastica", in 17a Conferenza Nazionale ASITA, Riva del Garda, novembre 2013, Federazione italiana delle Associazioni Scientifiche per le Informazioni Territoriali e Ambientali, pp. 799-806.

Lucchesi, F. (2002). "Nuove tecnologie per la descrizione e la rappresentazione del territorio", in M. Cusmano G., Città e insediamenti, Franco Angeli, Milano, pp. 68-79.

Lucchesi, F.; Maestro, F. Del; Dodero, A.; Flora, V.; Gabellieri, N.; Gesualdi, M.; Ghizzani Marcìa, F.; Giusti, B.; Grava, M.; Martinelli, A.; Marini, S.; Massarelli, M.; Rossi, M.; Tarchi, G.; Tofanelli, M.; Biagioli, G. (2014). "I nomi e luoghi. Densità toponomastica e struttura territoriale in Toscana tra XIX e XXI secolo", in 18a Conferenza Nazionale ASITA, Firenze, 14-16 ottobre 2014, ASITA - Federazione delle Associazioni Scientifiche per le Informazioni Territoriali e Ambientali, pp. 785-792.

Knowles, A.K. (2002). Past Time, Past Place: Gis for History, ESRI Inc., Redlands.

Panzieri, M. (2009). "Storiografia digitale e metafonti per la storia del territorio tra specificità disciplinari, standard web e dinamiche della rete", in Fonti, metafonti e GIS per l'indagine della struttura storica del territorio, Celid, Torino.

Mastrelli, C.A. (1998). "L'odonomastica nella legislazione italiana”, Rivista Italiana di Onomastica, IV, 2.

Pearson, A.W.; Collier, P. (2002). “Agricultural history with GIS”, in Knowles, A.K., Past Time, Past Place: GIS for history, ESRI Press, Redlands.

Repetti, E. (1833). Dizionario geografico fisico storico della Toscana contenente la descrizione di tutti i luoghi del Granducato, Ducato di Lucca, Garfagnana e Lunigiana, Firenze presso l'autore e editore coi tipi di Tofani A., Firenze, vol. 6, pp. 284-288.

Storti, M. (2007). Il paesaggio storico delle Cinque Terre. I "luoghi detti” condivisi, Firenze University Press, Firenze.

Sassoli, U. (2013). "I Catasti storici della Toscana e il progetto CASTORE", Rassegna degli Archivi di Stato, VII, pp. 113-119.

Sassoli, U. (2015). "The Historical Cadaster of Tuscany and the CASTORE Project", in Benedetti, B.; Farrugia, C.; Romiti, B.; Sipos, A., Cartography and cadastral maps Visions from the past for a vision of our future, Edizioni della Normale, pp. 71-81.

Trevisani, M. (2015). "Gli Open Geodata e la Regione Toscana”, GEOmedia, 6, pp. 36-40.

VV.AA. (1990). Dizionario di toponomastica. Storia e significato dei nomi geografici italiani, UTET, Torino.

Zamboni, A. (1994). "I nomi di luogo", in Storia della lingua italiana, vol. II, Scritto e parlato, a cura di Luca Serianni e Pietro Trifone, Torino, Einaudi. 
